\title{
Acute viral bronchiolitis in South Africa: Viral aetiology and clinical epidemiology
}

\author{
D A White, ${ }^{1}$ MB BCh, FC Paed (SA), MMed (Paed), Dip Allerg (SA), Cert Pulmonology (SA) Paed; \\ S A Madhi, ${ }^{2}$ MB BCh, MMed (Paed), FC Paed (SA), PhD; P Jeena, ${ }^{3}$ MB ChB, FC Paed (SA), Cert Pulmonology (SA) Paed; \\ H J Zar, ${ }^{4}$ MB BCh, FC Paed (SA), PhD; B M Morrow, ${ }^{5}$ PhD, BSc (Physio), PG Dipl (Health Research Ethics); \\ R Masekela, ${ }^{3}$ MB BCh, MMed (Paed), Cert Pulmonology (SA) Paed, Dip Allerg (SA), FCCP, PhD; \\ S Risenga ${ }^{6}$ MB ChB, FC Paed (SA), Dip Allerg (SA), Cert Pulmonology (SA) Paed; R J Green, ${ }^{7}$ PhD, DSc \\ ${ }^{1}$ Department of Paediatrics and Child Health, Faculty of Health Sciences, University of the Witwatersrand, Johannesburg, South Africa \\ ${ }^{2}$ Medical Research Council: Respiratory and Meningeal Pathogens Research Unit, University of the Witwatersrand, Johannesburg, South Africa \\ ${ }^{3}$ Department of Paediatrics and Child Health, Faculty of Health Sciences, University of KwaZulu-Natal, Durban, South Africa \\ ${ }^{4}$ Department of Paediatrics and Child Health, Red Cross War Memorial Children's Hospital, and MRC Unit on Child and Adolescent Health, \\ Faculty of Health Sciences, University of Cape Town, South Africa \\ ${ }^{5}$ Department of Paediatrics and Child Health, Faculty of Health Sciences, University of Cape Town, South Africa \\ ${ }^{6}$ Department of Pulmonology, Faculty of Health Sciences, University of Limpopo, Polokwane, and Pietersburg Hospital, South Africa \\ ${ }^{7}$ Department of Paediatrics and Child Health, Faculty of Health Sciences, University of Pretoria, South Africa
}

Corresponding author: D A White (debbie.white@wits.ac.za)

\begin{abstract}
Bronchiolitis is a viral-induced lower respiratory tract infection that occurs predominantly in children $<2$ years of age, particularly infants. Many viruses have been proven or attributed to cause bronchiolitis, including and most commonly the respiratory syncytial virus (RSV) and rhinovirus. RSV is responsible for more severe disease and complications (including hospitalisation) in bronchiolitis patients. Whereas bronchiolitis is exclusively due to respiratory viral infections, with little evidence of bacterial co-infection, the former could nevertheless predispose to superimposed bacterial infections. Although data support an interaction between RSV and pneumococcal superimposed infections, it should be noted that this specifically refers to children who are hospitalised with RSV-associated pneumonia, and not to children with bronchiolitis or milder outpatient RSV-associated illness. As such, empiric antibiotic treatment against pneumococcus in children with RSV-associated pneumonia is only warranted in cases of hospitalisation and when the clinical syndrome is more in keeping with pneumonia than uncomplicated bronchiolitis. In South Africa, the peak in the RSV season varies only slightly by province, with onset in February, and lasting until June. The important implication of these new seasonality findings is that where prophylaxis is possible, as in the case of RSV, it should be commenced in January of each year.
\end{abstract}

S Afr Med J 2016;106(5):443-445. DOI:10.7196/SAMJ.2016.v106i5.10444

\section{Definition}

Bronchiolitis is a viral-induced lower respiratory tract infection (LRTI) that occurs predominantly in children $<2$ years of age, particularly infants. ${ }^{[1]}$

\section{Causative organisms}

The most frequent cause of severe bronchiolitis is respiratory syncytial virus (RSV) infection, with other respiratory viruses (para-influenza virus (PIV), influenza virus, human metapneumovirus (hMPV) and measles virus) being less common or for which definitive attribution is yet to be established (e.g. rhinovirus (RV), bocavirus and coronavirus) (Table 1).

\section{Epidemiology}

Epidemiological studies on bronchiolitis often include all children presenting with physician-diagnosed LRTI, and may overestimate the true incidence of bronchiolitis alone.

In a prospective, hospital-based surveillance study of 8723 children under-5 with physi- cian-diagnosed LRTI, from five sites in four provinces in South Africa (SA), from 2009 to 2012 (severe acute respiratory illness (SARI) programme), the overall prevalence of respiratory viruses was $78 \%$. The viruses that were isolated included RV in $37 \%$, RSV in $26 \%$, adenovirus in $26 \%$, influenza virus in $7 \%$ and hMPV in 5\%. ${ }^{[2]}$ In 2009 and 2010, this surveillance study evaluated respiratory viruses by a 10 -plex real-time reverse-trans-

Table 1. Respiratory viruses associated with bronchiolitis

Respiratory syncytial virus

Rhinovirus

Adenovirus

Para-influenza virus (especially type 3 )

Influenza virus

Human metapneumovirus

Bocavirus

Coronavirus

Measles virus cription polymerase chain reaction (rRTPCR).${ }^{[3]}$ Respiratory viral co-infections among cases enrolled indicated $39.6 \%$ with single viral infections, while $17.4 \%$ had $\geq 2$ viral co-infections. ${ }^{[3]}$

\section{RV}

Many studies show RV to be associated with bronchiolitis; however, the high prevalence of RV in healthy asymptomatic children limits definitive attribution of its role in the pathogenesis of bronchiolitis. Early studies have suggested that oxygen saturation is generally not as low in children with RVassociated bronchiolitis as in those with RSV-associated bronchiolitis. ${ }^{[4]}$ However, more recent studies suggest that RV may be more sinister. ${ }^{[5]}$ All three types of RV have been identified in SA, although RV-A and $\mathrm{RV}-\mathrm{C}$ are more common than RV-B. RV is associated with symptomatic respiratory illness; however, there is no association between RV type and disease severity. ${ }^{[6]}$ RV-D has subsequently been identified. ${ }^{[7]}$ 


\section{RSV}

The two major RSV subgroups are A (RSV-A) and B (RSV-B), which are further characterised into several genotypes, based on antigenic and genetic variability of the second hypervariable region of the G-protein. Several genotypes can co-circulate in a single epidemic season, and different genotypes can dominate in consecutive seasons. Over a 15-year study period in SA, RSV-A and RSV-B genotypes evolved, resulting in replacement of all genotypes over the study period. ${ }^{[8]}$ Whereas genotype A virus, rather than RSV-B, has more frequently been associated with more severe disease, other studies have occasionally reported more severe disease with RSV-B than RSV-A. These differences could be related to the extent of community immunity to the specific genotype, with more severe disease observed in the presence of lower community immunity to that strain.

RSV is the most common cause of moderate or severe bronchiolitis and a leading cause of LRTI among young children. RSV-associated bronchiolitis occurs most frequently in infancy, being $2-3$ times higher than in children $>5$ years. Infection with RSV does not result in permanent or long-term immunity, as re-infections, albeit of lesser severity, are common and may be experienced throughout life. ${ }^{[9]} \mathrm{An}$ estimated 33.8 million new episodes of RSV-associated acute lower respiratory tract infection (ALRTI) occurred worldwide in 2005 in children under-5 (22\% of episodes), with at least 3.4 (2.8 - 4.3) million episodes necessitating hospital admission. An estimated 66000 199000 children under-5 died from RSV-associated ALRTI in 2005, with $99 \%$ of these deaths occurring in developing countries. ${ }^{[10]}$

In the SARI surveillance programme, the prevalence of RSV among 4293 LRTI hospitalisations in under-5 children was $27 \%$, including 863 of $1157(75 \%)<12$ months of age, of whom $637(74 \%)$ were $<6$ months old. Nine of 1153 children with RSV-associated ALRTI died (case fatality proportion $1 \%$ ), which was lower than the $2 \%$ (74 of 3122 ) of children in whom RSV was not identified $(p<0.001)$. Children admitted with RSV-associated ALRTI were younger than those who tested RSV negative (adjusted odds ratio (AOR) 2.8; 95\% confidence interval (CI) $2.1-3.7$ for age $<6$ months v. 23 - 59 months). Also, patients with RSV-associated ALRTI were more likely to present with cough (AOR 9.0; 95\% CI 5.5 - 14.9) and lower-chest retractions (AOR 1.4; 95\% CI 1.2 - 1.7) than children with non-RSV-associated ALRTI. ${ }^{[11]}$

The incidence of RSV-associated severe ALRTI in children from developing and developed countries (5.5/1 000 v. 5.6/1 000) is equal, but the case fatality rate is higher in the former $(2.1 \% \mathrm{v} .0 .3-0.7 \%) .{ }^{[10]}$ The case fatality rate for individual risk factors for RSV-associated disease among children with chronic lung disease is $3.5-23.0 \%$, congenital heart defects (CHDs) $2-37 \%$, nosocomial infection $0-12.2 \%$, intensive care unit admission $1.1-8.8 \%$, and prematurity $0.6-1.0 \%{ }^{[12]}$ The risk of mortality with RSV disease increases threefold among haemodynamically significant (complex disease with pulmonary hypertension) CHDs. ${ }^{[13]} \mathrm{HIV}$ is associated with a 2 - 3-fold greater risk of RSV disease and also with higher case fatality proportions ( $12 \%$ v. $2 \%$ in HIV-uninfected children). ${ }^{[11]}$

\section{Bacterial-viral interactions}

Whereas bronchiolitis is exclusively due to respiratory viral infections, with little evidence of bacterial co-infection, ${ }^{[14]}$ the former could nevertheless predispose to superimposed bacterial infections. ${ }^{[15]}$ These complicated cases of respiratory viral infections present with the more classic signs of pneumonia, including alveolar consolidation on chest radiographs, raised C-reactive protein $(\geq 40 \mathrm{mg} / \mathrm{dL})$, temperature $\geq 38^{\circ} \mathrm{C}$, crepitations and bronchial breathing on chest auscultation. The role of bacterial co- infections in children with a respiratory virus-associated pneumonia is frequently underemphasised owing to limited tools for diagnosing bacterial pneumonia, with blood culture sensitivity ranging from $3 \%$ to $18 \%$ for detecting pneumococcal pneumonia. ${ }^{[16]}$ However, epidemiological studies have identified a strong temporal association between some respiratory viruses and invasive pneumococcal disease. Included among these are studies on the temporal association of the influenza virus and RSV epidemics and invasive pneumococcal disease. ${ }^{[17]}$

Further evidence for this association was observed in an SA randomised controlled trial of an investigational 9-valent pneumococcal conjugate vaccine (PCV), in which children vaccinated with PCV had a $32 \%$ lower risk of being hospitalised for a viral-associated pneumonia compared with placebo recipients. ${ }^{[18]}$ This lower risk of respiratory virus-associated hospitalisation was evident for influenza virus, hMPV and RSV-associated pneumonia. ${ }^{[19]}$ The biological rationale for the reduction in respiratory virus-associated pneumonia among the PCV-vaccinated children in this study, was attributed to vaccination having prevented the superimposed vaccine-serotype pneumococcal co-infection, which would have led to progression to more severe disease - culminating in hospitalisation among the placebo recipients. Notably, there was no reduction in hospitalisation for bronchiolitis among the PCV9 vaccinated children, corroborating that pneumococcal co-infection was unlikely to have played a role in the pathogenesis of bronchiolitis.

The pathogenesis of increased susceptibility to pneumococcal infection following RSV infection in mice-model studies has been attributed to RSV G glycoprotein-binding penicillin-binding protein 1a increasing pneumococcal virulence owing to up-regulation of virulence genes, pneumococcal toxin and pneumolysin. This could lead to an increase in the inflammatory response and bacterial adherence to human ciliated epithelial cultures. ${ }^{[20,21]}$ This again is corroborated by studies in children with alveolar pneumonia associated with RSV or RV infection, among whom higher pneumococcal bacterial load was observed in the nasopharynx than in children with RSV or RV in the absence of alveolar consolidation. ${ }^{[22]}$ Observations from the SA study were recently corroborated by an ecological study in the USA, in which RSV and pneumococcal pneumonia were shown to share a distinctive spatiotemporal pattern, with RSV associated with a significant increase in the incidence of pneumococcal pneumonia in children $<1$ year of age (attributable percent (AP) 20.3\%; 95\% CI $17.4-25.1 \%$ ) and among children aged 1 - 2 years (AP 10.1\%; 95\% CI 7.6 - 13.9\%). Similarly, influenza was associated with an increase in pneumococcal pneumonia among children aged $1-2$ years (AP 3.2\%; 95\% CI $1.7-4.7 \%)$. After the introduction of PCV7 into the USA immunisation programme examining physician-coded diagnosis, the authors observed a decline in RSV-coded hospitalisations for children < 1 year old (AP $-18.0 \%$; 95\% CI -22.6 to $-13.1 \%$ for 2004/2005 - 2008/2009 v. 1997/1998 - 1999/2000). ${ }^{[23]}$

Although the abovementioned data support an interaction between RSV and pneumococcal superimposed infections, these specifically refer to children who are hospitalised with RSV-associated pneumonia and not to those with bronchiolitis or milder outpatient RSV-associated illness. As such, empiric antibiotic treatment against pneumococcus with RSV-associated pneumonia is only warranted in a child who is hospitalised and whose clinical syndrome is more in keeping with pneumonia than uncomplicated bronchiolitis.

\section{Seasonality}

There are several factors responsible for an outbreak of RSV infection. These include geographical locations (latitude and altitude) and climatic factors (temperature, barometric pressure, relative humidity, 
vapour tension, hours of light, precipitation, dewpoint). In most temperate regions, such as the USA and Europe, RSV outbreaks last an average of $3-4$ months, with a peak incidence during winter, although the exact timing of onset of the outbreak is uncertain. In tropical regions, RSV outbreaks are not distinctly related to season, but often occur during the hottest rainy season. In SA, the peak in RSV season varies slightly by province, with onset of the epidemic usually in KwaZulu-Natal in December - January, in Gauteng in February - March, and followed by the Western Cape in March. ${ }^{[24]}$

In 2009 and 2010, the SARI programme reported seasonality for RSV, influenza A and B viruses, enterovirus, hMPV, and PIV type 3. RSV circulation was evident from February to June, before the influenza season, which occurred from May to September. Peak activity for hMPV was observed between July and August and for PIV type 3 between September and November. Adenovirus and RV were identified perennially without any obvious peaks, while PIV types 1 and 2 were detected sporadically throughout the 2 years. ${ }^{[3]}$

The important implication of these new seasonality findings is that where prophylaxis is possible, as in the case of RSV, it should be commenced in January of each year.

\section{RSV in HIV-infected children}

RSV may be identified throughout the year in HIV-infected children because of prolonged shedding of the virus (up to 100 days post infection) compared with 5 - 7 days in HIV-uninfected children. ${ }^{[25]}$ Although HIVinfected children with RSV-associated ALRTI are at increased risk of hospitalisation and death, this could be due to greater susceptibility to Pneumocystis jirovecii pneumonia and other co-infections. The increased risk of RSV-associated ALRTI hospitalisation in HIV-infected children is greatest during infancy, but remains high even in the second, third, and fourth years of life. ${ }^{[11]}$

\section{Pathophysiology of $\mathbb{R S V}$ disease}

The viral infection starts in the upper respiratory tract and spreads to the lower tract within a few days, resulting in inflammation of the bronchiolar epithelium and oedema of the submucosa and adventitia. ${ }^{[26]}$ Plugs of sloughed, necrotic epithelium and fibrin and excessive mucus secretion add to airway obstruction, causing partial or total obstruction to airflow. ${ }^{[27]} \mathrm{A}$ 'ball-valve' mechanism can result in trapping of air distal to obstructed areas, with subsequent absorption, atelectasis, and a mismatch of pulmonary ventilation and perfusion that may lead to hypoxaemia. Smooth-muscle constriction seems to have a minor role in the pathological process, which may explain the limited benefit of bronchodilators observed in clinical studies. ${ }^{[26]}$ Although these mechanisms are known for RSV bronchiolitis, it is assumed that other viruses produce similar pathological conditions.

\section{References}

1. Wohl MEB. Bronchiolitis. In: Chernick V, Boat TF, Wilmot RW, Bush A, eds. Kendigs' Disorders of the Respiratory Tract in Children. Philadelphia: Saunders, 2006:423-432. DOI:10.1016/B978-0-7216-3695-5.50029-8

2. Cohen C, Walaza S, Moyes J, et al. Epidemiology of viral-associated acute lower respiratory tract infection among children $<5$ years of age in a high HIV prevalence setting, South Africa, 2009 - 2012. Pediatr Infect Dis I 2015;34:66-72. DOI:10.1097/INF.0000000000000478

3. Pretorius MA, Madhi SA, Cohen C, et al. Respiratory viral coinfections identified by a 10-plex real-time Pretorius MA, Madhi SA, Cohen C, et al. Respiratory viral coinfections identified by a 10-plex real-time
reverse-transcription polymerase chain reaction assay in patients hospitalized with severe acute respiratory reverse-transcription polymerase chain reaction assay in patients hospitalized with severe acute resp
illness - South Africa, 2009 - 2010. J Infect Dis 2012;206(S1):S159-S165. DOI:10.1093/infdis/iis538

illness - South Africa, $2009-2010$.J Infect Dis 2012;206(SI):S159-S165. DOI:10.1093/infdis/jis538
4. Matti K, Kotaniemi-Syrjänen A, Waris M, Vainionpää R, Reijonen TM. Rhinovirus-associated

Matti K, Kotaniemi-Syrjänen A, Waris M, Vainionpää R, Reijonen TM. Rhinovirus-associated
wheezing in infancy: Comparison with respiratory syncytial virus bronchiolitis. Pediatr Infect Dis J wheezing in infancy: Comparison with respiratory syncytia

2004;23:995-999. DOI:10.1097/01.inf.0000143642.72480.53
Morrow BM, Feldman C, Green RJ. Acute viral bronchiolitis in South Africa: Intensive care managemen for severe disease. S Afr Med J 2016;106(5):446-448. DOI:10.7196/SAMJ.2016.v106i5.10436

6. Pretorius MA, Tempia S, Treurnicht FK, et al. Genetic diversity and molecular epidemiology of human rhinoviruses in South Africa. Influenza Other Respir Virus 2014;8(5):567-573. DOI:10.1111/irv.12264

. Palmenberg AC, Spiro D, Kuzmickas R, et al. Sequencing and analyses of all known human rhinovirus genomes reveal structure and evolution. Science 2009;324(5923):55-59. DOI:10.1126/science.1165557

Pretorius MA, van Niekerk S, Tempia S, et al. Replacement and positive evolution of subtype A and B respiratory syncytial virus G-protein genotypes from 1997 - 2012 in South Africa. J Infect Dis 2013;208(S3):S227-S237. DOI:10.1093/infdis/jit477

9. American Academy of Pediatrics, Subcommittee on Diagnosis and Management of Bronchiolitis. Diagnosis American Academy of Pediatrics, Subcommittee on Diagnosis and Management of Bronchiolitis. Dag
and Management of Bronchiolitis. Pediatrics 2006;118(4):1774-1793. DOI:10.1542/peds.2006-2223

10. Nair H, Nokes DJ, Gessner BD, Dherani M, Madhi SA, Singleton RJ. Global burden of acute lower Nair H, Nokes DJ, Gessner BD, Dherani M, Madhi SA, Singleton RJ. Global burden of acute lower
respiratory infections due to respiratory syncytial virus in young children: A systematic review and meta-analysis. Lancet 2010:375:1545-1555. DOI-10.1016/S0140-6736(10)60206-1

11. Moyes J, Cohen C, Pretorius M, et al. Epidemiology of respiratory syncytial virus-associated acute lower respiratory tract infection hospitalizations among HIV-infected and HIV-uninfected South African children, 2010 - 2011. J Infect Dis 2013;208(S3):S217-S226. DOI:10.1093/infdis/jit479

12. Welliver RC Sr, Checchia PA, Bauman JH, Fernandes AW, Mahadevia PJ, Hall CB. Fatality rates in published reports of RSV hospitalizations among high-risk and otherwise healthy children. Curr Med Res Opin 2010;26(9):2175-2181. DOI:10.1185/03007995.2010.505126

13. Altman CA, Englund JA, Demmler G, et al. Respiratory syncytial virus in patients with congenital heart disease: A contemporary look at epidemiology and success of preoperative screening. Pediatr Cardiol 2000;21(5):433-438. DOI:10.1007/s002460010103

14. Hall CB, Powell KR, Schnabel KC, Gala CL, Pincus PH. Risk of secondary bacterial infection in infants hospitalized with respiratory syncytial viral infection. J Pediatr 1988;113(2):266-271. DOI:10.1016/S0022-3476(88)80263-4

15. Moore DP, Dagan R, Madhi SA. Respiratory viral and pneumococcal coinfection of the respiratory Moore DP, Dagan R, Madhi SA. Respiratory viral and pneumococcal coinfection of the respiratory
tract: Implications of pneumococcal vaccination. Expert Rev Respir Med 2012;6(4):451-465. tract: Implications of

16. Madhi SA, Kuwanda L, Cutland C, Klugman KP. The impact of a 9-valent pneumococcal conjugate vaccine on the public health burden of pneumonia in HIV-infected and -uninfected children. Clin Infect Dis 2005;40(10):1511-1518. DOI:10.1086/429828

17. Talbot TR, Poehling KA, Hartert TV, et al. Seasonality of invasive pneumococcal disease: Temporal relation to documented influenza and respiratory syncytial viral circulation. Am J Med 2005;118(3):285-291. DOI:10.1016/j.amjmed.2004.09.016

18. Madhi SA, Klugman KP. A role for Streptococcus pneumoniae in virus-associated pneumonia. Nat Med 2004;10(8):811-813. DOI:10.1038/nm1077

9. Madhi SA, Ludewick H, Kuwanda L, et al. Pneumococcal coinfection with human metapneumovirus. J Infect Dis 2006;193(9):1236-1243. DOI:10.1086/503053

20. Smith CM, Sandrini S, Datta S, et al. Respiratory syncytial virus increases the virulence of Streptococcus pneumoniae by binding to penicillin binding protein la. A new paradigm in respiratory infection. Am J Respir Crit Care Med 2014;190(2):196-207. DOI:10.1164/rccm.201311-21100C

21. Hament JM, Aerts PC, Fleer A, et al. Enhanced adherence of Streptococcus pneumoniae to Hament JM, Aerts PC, Fleer A, et al. Enhanced adherence of Streptococcus pneumoniae to
human epithelial cells infected with respiratory syncytial virus. Pediatr Res 2004;55(6):972-978. DOI:10.1203/01.PDR.0000127431.11750.D9

22. Esposito S, Marchese A, Tozzi AE, et al. DNA bacterial load in children with bacteremic pneumococcal community-acquired pneumonia. Eur J Clin Microbiol Infect Dis 2013;32(7):877-881. DOI:10.1007/ s10096-013-1821-0

23. Weinberger DM, Klugman KP, Steiner CA, Simonsen L, Viboud C. Association between respiratory syncytial virus activity and pneumococcal disease in infants: A time series analysis of US hospitalization data. PLoS Med 2015;12(1):e1001776. DOI:10.1371/journal.pmed.1001776

24. Green RJ, Zar HJ, Jeena PM, et al. South African guideline for the diagnosis, management and prevention of acute viral bronchiolitis in children. S Afr Med J 2010;100(5):320-325. DOI:10.7196/SAMJ.4016

25. King JC, Burke AR, Clemens JD, et al. Respiratory syncytial virus illnesses in human immunodeficiency virus- and non-infected children. Pediatr Infect Dis I 1993;12:733-739. DOI:10.1097/00006454-199309000-00006

26. Zorc JJ, Hall CB. Bronchiolitis: Recent evidence on diagnosis and management. Pediatrics 2010;125:342-349. DOI:10.1542/peds.2009-2092

27. McNamara PS, Smyth RL.The pathogenesis of respiratory syncytial virus disease in childhood. Br Med Bull 2002;61(1):13-28. DOI:10.1093/bmb/61.1.13 